\title{
IDENTIFICATION OF MASS OF TISSUE GROWTH IN BRAIN REGION UNDER THE FRAMEWORK OF IMAGE SEGMENTATION
}

\author{
Kumar Gaurav Verma ${ }^{1}$, Somsubhra Gupta ${ }^{2}$ and Debasree Mitra ${ }^{3}$
}

\begin{abstract}
Magnetic resonance imaging (MRI) or magnetic resonance tomography (MRT) is a medical imaging technique used in radiology to image the anatomy and the physiological processes of the body in both health and disease. There are MRI scanners which uses strong magnetic field and field gradient to form images of the body. To detect any type of growth in the brain region we can use this technique. Growths can me natural and unnatural. In this project we propose an image segmentation method to identify or detect any unnatural growth in brain region taking input as a Magnetic Resonance Imaging (MRI) image of the brain which has some unnatural growth. Whether the detected growth is tumor or cancer, this will be our future scope of the paper.

Keywords - MRI ,Unnatural Growth, Image Segmentation, Malignancy, Watershed algorithm, Image complement, Image Filtration, Distance transformation, Edge Detection.
\end{abstract}

\section{INTRODUCTION}

Malicious is the adjective based on the noun malice which means the desire to harm. Malicious tumor represents any type of cancer which has the ability to consume and destroy neighbor tissues. Any unnatural growth under the brain region can malignant. Brain tumors are non malignant growth. They occur due to rapidly growing of the bad cells or tissues at the same place. There is no any age limitation to occur this problem but once it occur it must be removed within time otherwise it will subjected to the death of the person suffering from the same, so study of unnatural growth specially tumors and cancers are very important and there detection with simple procedures which take less time and provide more accuracy and is cost effective is the most important part.

\section{IMAGE SEGMENTATION}

Image segmentation is the process of subdividing an image into multiple or constituent segments. The level of segmentation is based on the application. Image segmentation is typically used to locate objects and boundaries i.e. lines, curves, etc in images. Image segmentation algorithms generally are based on one of two basic properties of intensity values as for example one is discontinuity and other property is similarity. The result of image segmentation is a set of segments that collectively cover the entire image, or a set of contours extracted from the image. Image registration is the process of transforming different sets of data into one coordinate system.

A. Needs of Image Segmentation

The following are the essential steps for image segmentation:

- Essential step in image analysis.

- Essential step in object representation

- Essential step in objet visualization

- Segmentation bridges the gap between low-level image processing and high level image processing, etc.

B. Applications of Image Segmentation

The following are the some applications of image segmentation:

- Industrial inspection.

- In optical character recognition(OCR)

- In medical science like detection of brain tumors, measurement of bone tissues, etc.

\footnotetext{
${ }^{1}$ Department of Computer Science and Engineering, JIS College of Engineering, West Bengal, INDIA, Email: depgaurav.verma786@gmail.com

2 Department of Computer Science and Engineering and Information Technology, JIS College of Engineering, West Bengal, INDIA, Email: gsomubhra@gmail.com

${ }^{3}$ Department of Computer Science and Engineering, JIS College of Engineering, West Bengal, INDIA, Email: debasree.mitra2005@gmail.com
} 


\section{Image Segmentation Technique}

The existing techniques for image segmentations are presented in the following articles

\section{Discontinuity based image segmentation:}

It includes three types of detections:
a) Point detection
b) Edge detection
c) Line detection

The most common way to look for discontinuities is to run a mask through the image. As for example a $3 \mathrm{X} 3$ mask is given below, this procedure involves computing the sum of products of the coefficients with the gray levels contained in the region encompassed by the mask. That is, the response of the mask at any point in the image is given by

$$
R=\sum_{i=1}^{l} \sum_{j=1}^{l} w_{i, j} f(x+i, y+j)
$$

Table 1. Discontinuity based image segmentation

\begin{tabular}{|c|c|c|}
\hline $\mathrm{W}_{1}$ & $\mathrm{~W}_{2}$ & $\mathrm{~W}_{3}$ \\
$-1,-1$ & $-1,0$ & $-1,1$ \\
\hline $\mathrm{W}_{4}$ & $\mathrm{~W}_{5}$ & $\mathrm{~W} 6$ \\
$0,-1$ & 0,0 & 0,1 \\
\hline $\mathrm{W}_{7}$ & $\mathrm{~W}_{8}$ & $\mathrm{~W}_{9}$ \\
$1,-1$ & 1,0 & 1,1 \\
\hline
\end{tabular}

\section{A. Point Detection}

For the point detection, we consider the modulus of above value of $\mathrm{R}$ as greater than the non negative Threshold value

$$
|\mathrm{R}|>=\mathrm{T}
$$

\section{B. Line Detection}

Line detection is also based on the abrupt change in the intensity of an image and additionally it reflects the discontinuity in image. in case of line detection the following mask is used:

Table 2. Segmentation matrices with various slope



a) Horizontal

\begin{tabular}{|l|l|l|}
\hline-1 & -1 & 2 \\
\hline-1 & 2 & -1 \\
\hline 2 & -1 & -1 \\
\hline
\end{tabular}

b) $+45^{\circ}$

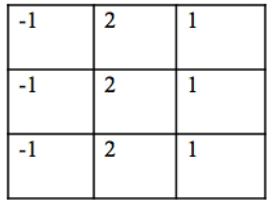

c) Vertical

\begin{tabular}{|l|l|l|}
\hline 2 & -1 & -1 \\
\hline-1 & 2 & -1 \\
\hline-1 & -1 & 2 \\
\hline
\end{tabular}

d) $-45^{\circ}$

If the first mask were moved around an image, it would respond more strongly to lines i.e. one pixel thick, oriented horizontally with a constant background, the maximum response would result when the line passed through the middle row of the mask. This is easily verified by sketching a simple array of 1's with a line of a different gray level say 5's, running horizontally through the array. The coefficients in each mask sum to zero, indicating a zero response from the masks in areas of constant gray level. Suppose, that the four masks are run individually through an image. If, at a certain point in the image, $\left|R_{i}\right|>\left|R_{j}\right|$, for all $i \neq j$, then the particular point is more likely to be contained in a line which is in the direction of Mask $\mathrm{i}_{\mathrm{i}}$

\section{Edge Detection}

Edge detection is a most common approach to segmentation which detecting meaningful discontinuities in gray level. An edge is boundary between two regions having distinct intensity level. There are many ways to perform edge detection. However, the most may be grouped into two categories, gradient and Laplacian. The gradient method detects the edges by looking for the maximum and minimum in the first derivative of the image. The Laplacian method searches for zerocrossings in the second derivative of the image to find edges.

\section{Canny operator:}

It is such edge detector which uses a multi-stage algorithm to detect a wide range of edges in images. This process involves four processes. First a Gaussian blur is applied to clear any speckles and free the image of noise. Then a gradient operator is applied for obtaining the gradients' intensity and direction. Non-maximum suppression determines if the pixel is a better candidate for an edge than its neighbour's.At last Hysteresis thresholding finds where edges begin and end. 
Similarity based image segmentation:

It includes Threshold operation, region growing region splitting and merging.

\section{Thresholding:}

Consider an image that contains two types of regions R1 and R2 and the distinctness of the regions is reflected very clearly. Suppose there exists a threshold $t$ such that feature values of all pixels that actually belong to regions of first type are less than or equal to $t$ and gray values of all pixels that actually belong to regions of the second type are greater than $t$. In this case, the segmented image is obtained as

$$
\begin{aligned}
& \mathrm{b}(\mathrm{r}, \mathrm{c})=1 \text { if } \mathrm{p}(\mathrm{r}, \mathrm{c}) \quad<=\mathrm{t} \text { (background) } \\
& 0 \text { if } \mathrm{p}(\mathrm{r}, \mathrm{c}) \quad>\mathrm{t}(\mathrm{object}) \\
& \text { In which } \mathrm{p}(\mathrm{r}, \mathrm{c}) \text { is the feature value at pixel }(\mathrm{r}, \mathrm{c}) .
\end{aligned}
$$

\section{Region Growing}

Region growing is a simple region-based image segmentation method. It is also classified as a pixel-based image segmentation method since it involves the selection of initial seed points. This approach to segmentation examines neighboring pixels of initial seed points and determines whether the pixel neighbors should be added to the region. The process is iterated on, in the same manner as general data clustering algorithms. The main goal of segmentation is to partition an image into regions. Region-based segmentation is a technique for determining the region directly.

The basic formulation is:

(a) $\cup n i=1 R_{i}=R$.

(b) $\mathrm{Ri}$ is a connected region, $\mathrm{i}=1,2, \ldots, \mathrm{n}$

(c) $\mathrm{R}_{\mathrm{i}} \cap \mathrm{R}_{\mathrm{i}}=\varnothing$ for all $\mathrm{i}=1,2, \ldots, \mathrm{n}$.

(d) $P\left(R_{i}\right)=$ TRUE for $i=1,2, \ldots, n$.

(e) $P\left(R_{i} \cup R_{i}\right)=F A L S E$ for any adjacent region $R_{i}$ and $R_{i}$.

$P\left(R_{i}\right)$ is a logical predicate defined over the points in set $R_{i}$ and $\varnothing$ is the null set.

\section{Region Splitting}

In region splitting approach we have to consider the homogeneity property over a rectangular region. If the gray levels present in the region do not satisfy the property, we divide the region into four equal quadrants. If the property is satisfied, we leave the region as it is. This is done recursively until all the regions satisfy the property.

In terms of graph theory, let us call a region a node. The node is split into four children if the node does not satisfy the given property; otherwise the node is left unaffected. The former node is called a parent node and the later a leaf node. This method is applicable to images whose number of rows and number of columns are some integer power of 2 .We start the method taking the whole image. That means the image is taken as the root node and a quad tree is formed where each leaf node represents a rectangular homogeneous region.

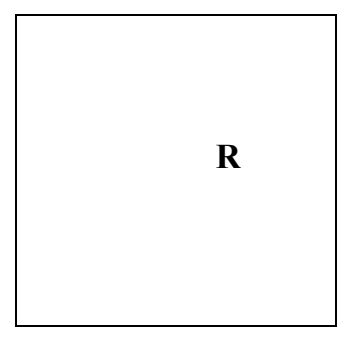

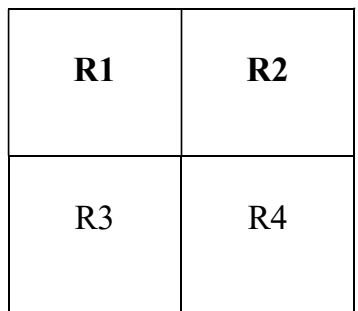

Figure1: Rectangular Region Splitting

\begin{tabular}{|c|c|c|}
\hline \multirow{2}{*}{ R1 } & R21 & R22 \\
\cline { 2 - 3 } & R23 & R24 \\
\hline \multirow{2}{*}{ R3 } & R31 & R32 \\
\cline { 2 - 3 } & R33 & R34 \\
\hline
\end{tabular}

\section{Region Merging}

This method is exactly opposite to the region splitting method. Like region splitting, this method is only applicable for that image having number of rows and number of columns is some integer power of 2.Here we start from pixel level and we consider each of them a homogenous region. At any level of merging, we check if four adjacent homogeneous regions arranged in a $2 \mathrm{X} 2$ fashion together satisfies the homogeneity property. If yes, we merge those regions to a single homogeneous region; otherwise the regions are left as they are. Hence in terms of graph theory, child nodes are removed if the parent node satisfies the homogeneity property; otherwise child nodes are declared as leaf node. We repeat this operation recursively until there are no more regions that can be merged. 


\begin{tabular}{|l|l|l|l|l|l|}
\hline R11 & R12 & R21 & R22 \\
\hline R13 & R14 & R23 & R24 \\
\hline R31 & R32 & R41 & R42 \\
R33 & R34 & R43 & R44 & R1 & R2 \\
\cline { 3 - 5 } & & R3 & R4 \\
\hline
\end{tabular}$\quad$\begin{tabular}{|l} 
\\
\hline
\end{tabular}

\section{IMAGE COMPLETION}

Image complementation is the technique in which a zeros of a binary image becomes ones and ones becomes zeros, black and white are reversed. In the complement of an intensity or RGB image, each pixel value is subtracted from the maximum pixel value supported by the class and the difference is used a s pixel value in the output image. In the output image, dark areas become light and light areas become darker.

\section{Image Filtration}

Filtering is a technique for modifying or enhancing an image. It is used to emphasize certain features or remove other features. Image processing operations implemented with filtering include smoothing, sharpening, and edge enhancement. Filtering is a neighborhood operation, in which the value of any given pixel in the output image is determined by applying some algorithm to the values of the pixels in the neighborhood of the corresponding input pixel. A pixel's neighborhood is some set of pixels, defined by their locations relative to that pixel. Linear filtering is filtering in which the value of an output pixel is a linear combination of the values of the pixels in the input pixel's neighborhood.

\section{Watershed Algorithm}

Watershed transform is the technique which is commonly used in image segmentation. It is now being recognized as a powerful method used in image segmentation due to its many advantages such as simplicity, speed and complete division of the image. Watershed transform or Watershed Algorithm is based on grey-scale morphology. It is classified as a region-based segmentation approach. Even when the target regions having low contrast and week boundaries, watershed transformation can provide closed contours.

When a landscape or topographic relief is flooded with water, the divide lines of the domains of rain falling over the regions forms the watersheds. Intuitively, a drop of water falling on a topographic relief flows towards the "nearest" minimum. The "nearest" minimum is that minimum which lies at the end of the path of steepest descent. In terms of topography, this occurs if the point lies in the catchment basin of that minimum.

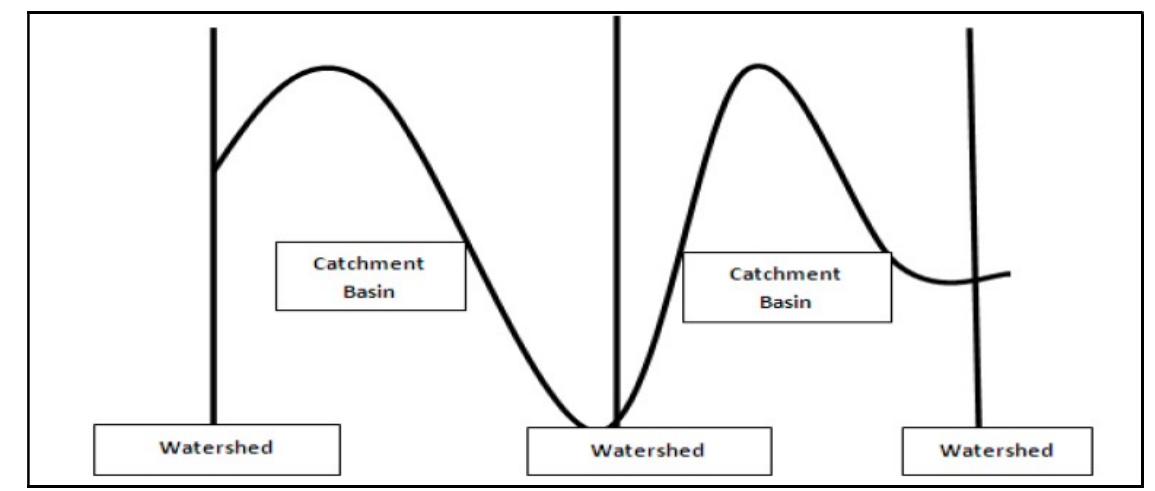

Figure 3: Watershed segmentation-local minima yield catchment basins; local maxima define the watershed lines

An alternative approach is to imagine the landscape being immersed in a lake in which holes are pierced in the local minima is called the catchment basin. Water will be filled up at these starting local minima and at points where water coming from different basins would meet and dams will be built. When the water level reaches the highest peak in the landscape the process is stopped. As a result, the landscape is partitioned into regions or basins separated by dams, called watershed lines or simply watersheds. When simulating this process for image segmentation, two approaches may be used: either one first finds 
basins, then watersheds by taking a set complement; or one computes a complete partition of the image into basins, and subsequently finds the watersheds by boundary detection.

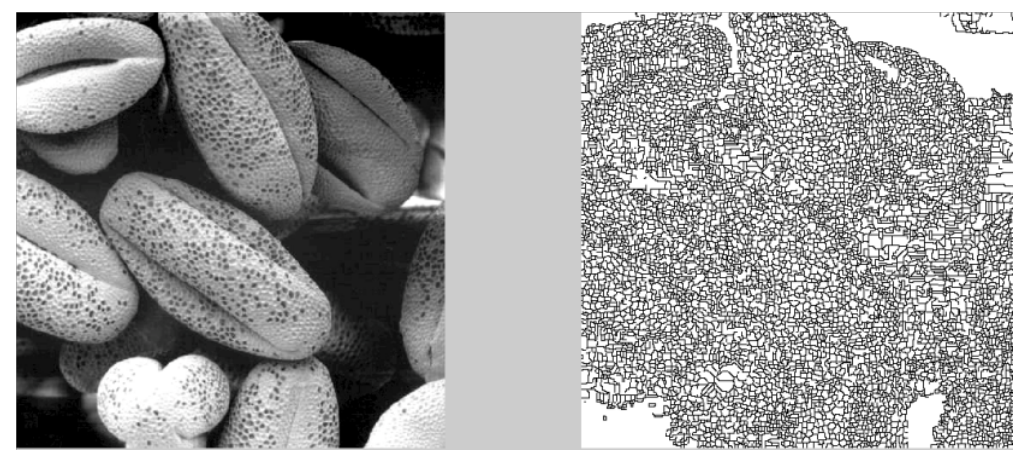

Figure 4: An Example of Watershed Conversion

\section{MethodologicAL ASPECTS}

In this work, an important approach on watershed algorithm has been investigated for obtaining better result in image segmentation. This algorithm is developed for getting better segmented image, which is less over-segmented.

In this proposed approach, firstly a normal brain MRI image is chosen, and converted to grey-scale image. Secondly we apply canny edge detector operator for edge detection. Thirdly image complement is obtained from the previous resulted image. And finally watershed transformation is applied to the image to get the desired result and the values for different parameters like golden number, Eulers number and Eigen value is calculated the again we take an image which is defected and again we apply the same procedure to the image and obtained the values for the same parameters that we have calculated earlier and compared the two vales that obtained and obtained the desired result.

A flow chart of the process is given below:

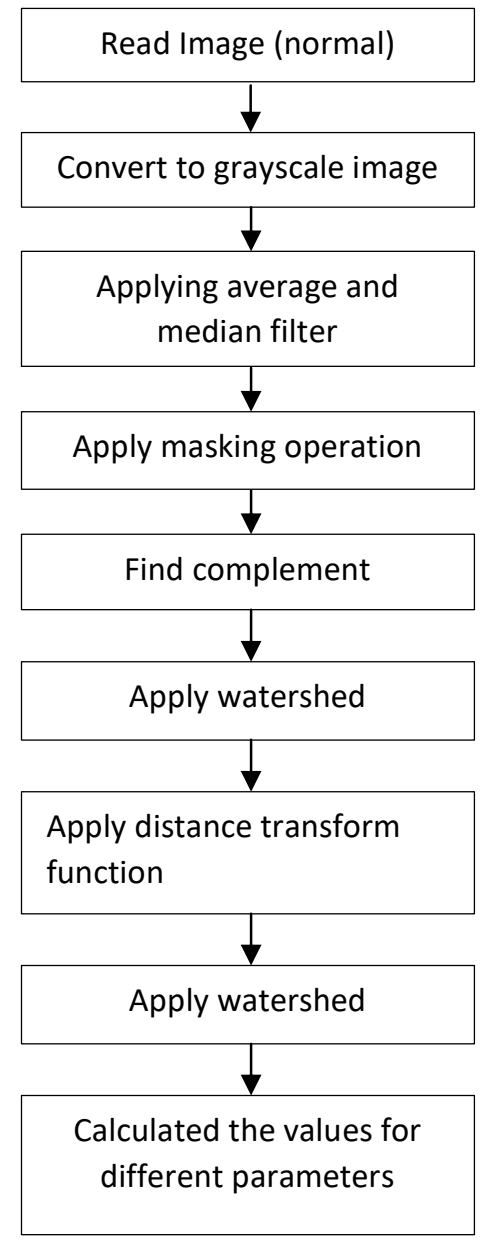

Figure 5: Pictorial representation 


\section{RESULT AND DISCUSSIONS}

The analyzed images after employing the methodology, has been sequentially presented below:

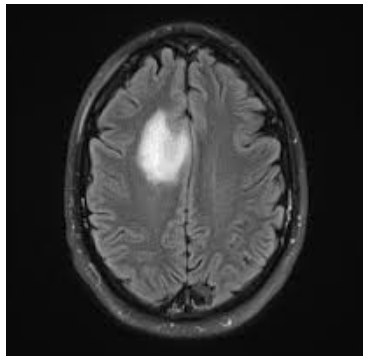

Read Image

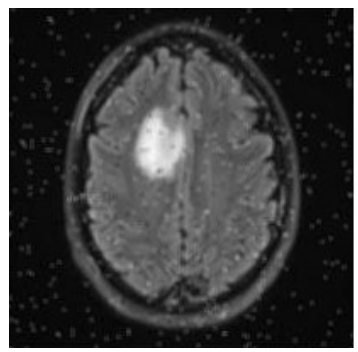

Applying Average filter

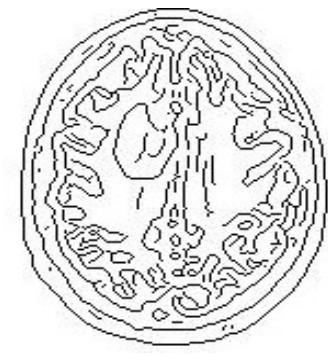

Image Complement

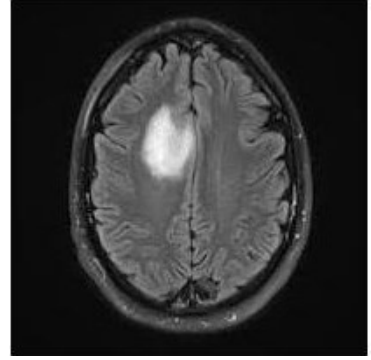

Converted Gray scale Image

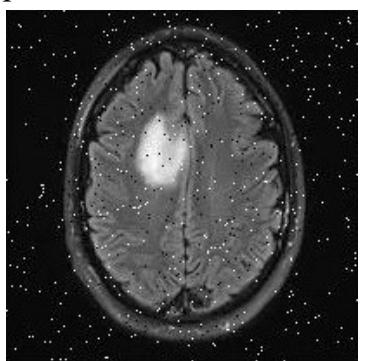

Noise Added Image

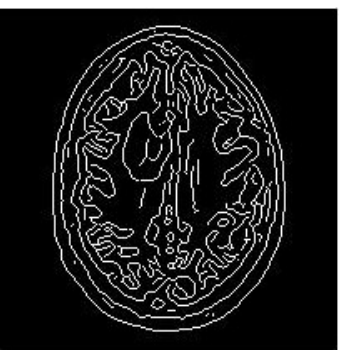

Canny 1 degree operator

Applying Median filter

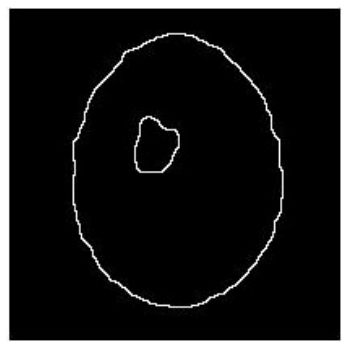

After Distance transformation

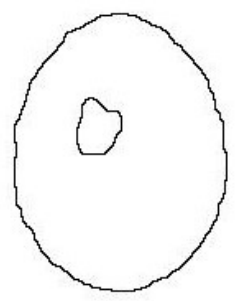

Applying Watershed

Figure 6: Step-wise obtained brain-tumor region images

From the result obtained above, it is evident that the proposed methodology, the region of interested can be admissibly recognized with desired accuracy through the proposed methodology pattern recognition through digital image processing.

\section{CONCLuSION}

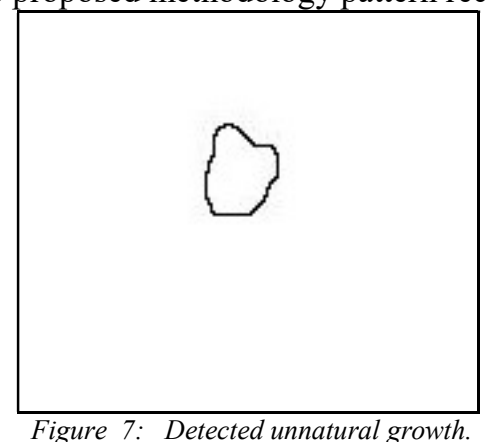

Brain tumor, a mass of tissue that grows out of control is one of the major causes for the increase in mortality among children and adults. Image segmentation is an important part of image processing. In this paper we have successfully introduced a new approach to image segmentation technique which is based on Watershed Algorithm using Distance Transform. . Hence, it has 
been concluded that the new approach that we have discussed in this paper has certainly shown a difference to detect region of interest of the images. In future we can also perform abnormal growth detection by Support Vector Machine model, principal component analysis and also by Hidden Markov Model. The above mentioned method has huge potential in the field of biomedical image processing domain. In this paper we have discussed only how detect growth of mass of tissue in brain region and till we have not found the malignancy of the mass of tissue growth . The malignancy can be found by neuro fuzzy segmentation process.

\section{REFERENCES}

[1] A.K.Jain, Fundamentals of digital image processing, Second Edition, Prentice Hall, 2002.

[2] K. Parvati, B. S. PrakasaRao and M. Mariya Das, "Image Segmentation Using Gray-Scale Morphology and Marker-ControlledWatershed Transformation", Discrete Dynamics in Nature and Society,vol. 2008, pp. 1-8, 2008.

[3] Gonzalez \& Woods, Digital Image Processing, 3rd edition, Prentice Hall India, 2008.

[4] S. Beucher, "Watershed, hierarchical segmentation and water fall algorithm," in Mathematical Morphology and Its Applications to Image Processing, Dordrecht, The Netherlands: Kluwer, 1994, pp. 69-76.

[5] Beucher, S., and Lantuejoul, C, "Use of watersheds in contour detection", In Proc. International Work-shop on Image Processing, Real-Time Edge and Motion Detection/Estimation, Rennes, pp.17-21, France, September 1979.

[6] Vicent L. Solille P, Watershed in digital spaces, “An efficient algorithm based immersion simulations," IEEE TransectionsPAMI,vol. 13.no6. pp. 538$598,1991$.

[7] C. Riddell, p. Brigger, R. E. Carson and S. L. Bacharach, "The watershed algorithm: a method to segment noisy PET transmission images," IEEE Trans. Nucl. Sci., vol. 46, no. 3, pp. 713-719, Mar, 1999.

[8] Rafael C. Gonzalez, Richard E. Woods, Steven L. Eddins, "Digital Image Processing Using MATLAB," Second Edition, Gatesmark Publishing, 2009.

[9] S. Thilagamani, N.Shanthi, "A Novel Recursive Clustering Algorithm for Image Oversegmentation", European Journal of Scientific Research, Vol.52, No.3, pp.430-436, 2011.

[10] F. Zou, Y. Zheng, Z. Zhou, and K. Agyepong, "Gradient vector flow field and mass region extraction in digital mammograms," International Symposium Computer-Based Medical Systems, pp. 41-43, June 2008.

[11] H. Zhang and G. Li, "Application in stomach epidermis tumors segmentation by GVF snake model," International Seminar on Future BioMedical Information Engineering, pp. 453-456, 2008.

[12] M. Kass, A. Witkin, and D. Trezopoulos, "Snakes: Active contour models," International Journal of Computer Vision, vol. 1, no. 4, pp. 321-331, 1987.

[13] C. Davatzikos, "Spatial transformation and registration of brain images using elastically deformable models," Comput. Vis. Image Understand., vol. 66, no. 2, pp. 207-222, 1997.

[14] D.Mitra,R.Barik,S.Roy,S.Bhattacharyya “A Survey on Image Segmentation and Image Registration” ,ACEEE-CPS, International Conference on Computing,Communication \& Manufacturing, ISBN: 978-0-9940194-0-0,Pages 61-69

[15] D.Mitra,R.Barik,S.Roy,S.Bhattacharyya“"Cumulative Measurement of Image Entropy on Different Mathematical Morphological Operation”,ACEEECPS, International Conference on Computing,Communication \& Manufacturing, ISBN: 978-0-9940194-0-0,Pages 35-39

[16] D Mitra and K Gaurav Verma “Information Processing using Multilevel Masking to Image Segmentation" International Journal of Computer Applications 141(3):1-6, May 2016.ISBN : 973-93-80893-05-3, doi:10.5120/ijca2016909567

[17] N. R. Pal and S. K. Pal , “A review on image segmentation techniques," Pattern Recognition, vol. 26, no. 9, pp. 1277-1294, 1993

[18] L. P. Clarke, R. P. Velthuizen, M. A. Camacho, J. J. Heine, M. aidyanathan, L. O. Hall, R. W. Thatcher, and M. L. Silbiger, "MRI segmentation: methods and applications," Magn Reson Imaging, vol. 13, no. 3, pp. 343-68, 1995.

[19] M. B. Suchendra, K. B. Jean, and S. B. Minsoo, "Multiscale image segmentation using a hierarchical self-organizing map,"Neurocomputing, vol. 14, pp. 241-272, 1997.

[20] L. Wei, Y. Yang, R. M. Nishikawa, Y. Jiang, "A Study on Several Machine- Learning Methods for Classification of Malignant and Benign Clustered Microcalcifications", IEEE Transactions on Medical Imaging, pp. 1-10, Jan 2005

[21] M. Pontil and A. Verri, "Support vector machines for 3-D object recognition", IEEE Transactions on Pattern Analysis and Machine Intelligence, vol. 20, pp. 637-646, June 1998

[22] R. Chandrasekhar, and Y. Attikiouzel, Y. Automatic, "Breast Border egmentation by Background Modeling and Subtraction", in: M.J. Yaffe (Ed.), Proceedings of the 5th International Workshop on Digital Mammography (IWDM), Medical Physics Publishing, Toronto, Canada, 2000, pp. 560-565.

[23] J.L. Semmlow, A. Shadagopappan, L.V. Ackerman, W. Hand, and F.S. Alcorn, "A Fully Automated System for Screening Xeromammograms", Computers and Biomedical Research, vol. 13, pp. 350-362, 1980

[24] M. Masek, Y. Attikiouzel, and C.J.S. deSilva, "Skin-air Interface Extraction from Mammograms Using an Automatic Local Thresholding Algorithm", in Proc. of the 15th Biennial International Conference Biosignal, pp. 204-206, 2000. 